\title{
ECG-Gated ICUS Image Acquisition Combined with a Semi-Automated Contour Detection Provides Accurate Analysis of Vessel Dimensions
}

\author{
$\mathrm{N}$ Bruining, $\mathrm{C}$ von Birgelen, M T Mallus, P J de Feyter, E de Vrey, \\ W Li, F Prati, P W Serruys, J R T C Roelandt \\ Erasmus University/University Hospital Rotterdam Dijkzigt, Thoraxcenter, \\ Rotterdam, The Netherlands
}

\begin{abstract}
At present most systems used for three-dimensional (3D) reconstruction's of two-dimensional Intracoronary ultrasound (ICUS) images are based on an image acquisition with a pull-back device which withdraws the catheter with a constant speed, not taking account of cardiac motion and coronary dynamics/pulsation. Cyclic changes of the vessel dimensions and the movement of the catheter inside the vessel result in artifacts and inaccuracies of quantitative analysis. Furthermore, most systems in use for this kind of analysis applications store the ICUS image data on video tape for off-line analysis, which results in quality loss.

To overcome these limitations and to achieve a rapid on-line analysis without quality loss resulting from unnecessary $A / D$-conversions, ECG-gated acquisition and direct digitisation of ICUS images was performed on-line.
\end{abstract}

\section{Introduction}

Intracoronary ultrasound (ICUS) provides highresolution cross-sectional imaging of the vessel wall which is increasingly used during diagnostic cardiac catheterization and as a guiding tool during interventional procedures. However, it is hard to mentally conceptualise a tomographic data set of 2-D parallel sliced ICUS images into a 3-D structure. Furthermore single measurements, often performed manually, by example proximal, within and distal of the stenoses provides limited accurate analysis results. At present most $3-\mathrm{D}$ and analysis systems commercially available are based on an image acquisition with a pull-back device which withdraws the ICUS catheter at an uniform speed, not taking account of the cardiac motion and coronary dynamics/pulsation. Artifacts and inaccurate measurements of the tomographic data set may result from the systolic-diastolic changes of the vascular dimensions and the cyclic movement of the catheter [1]. A secondary problem is that most systems store the ICUS image data on video tape for off-line analysis which results in severe quality loss [2].

Automated ECG-gated ICUS image acquisition combined with 3-D rendering and Semi-automated contour detection software allows to overcome these limitations.

\subsection{Background}

In recent years several methods have been developed to acquire tomographic ICUS image data sets and to perform 3-D reconstruction's and 3-D vessel analysis:

- Manual pull-back.

- Manual pull-back with the ICUS catheter shaft placed in a displacement sensing device.

- Motorised pull-back by an uniform velocity motor, applying a speed of $1-0.2 \mathrm{~mm} / \mathrm{sec}$ [3].

- Motorised pull-back by an uniform velocity motor and use of an ECG-labelling device so that only one phase during the $\mathrm{R}-\mathrm{R}$ interval is acquired at a continuous speed of $0.2 \mathrm{~mm} / \mathrm{sec}$ ("Pseudo-Gating") [4].

- Motorised ECG-gated pull-back using a stepping motor.

The technique described in this paper is based on analysis on ICUS image data sets which are acquired with a motorised ECG-gated pull-back device.

\section{ICUS Acquisition Set-up}

A scheme of the set-up of the 3-D reconstruction system in the catheterization laboratory is shown in figure 1 . The ultrasound examinations have been performed using a $2.9 \mathrm{~F}$ MicroView $30 \mathrm{MHz}$ mechanical rotating element catheter (CVIS, Sunnyvale, CA, USA) and the echo units Insight III and HP Sonos (Hewllet-Packard,Andover, MA, USA).

The catheter configuration (external echo-transparent sheath with an independent imaging cable inside) 


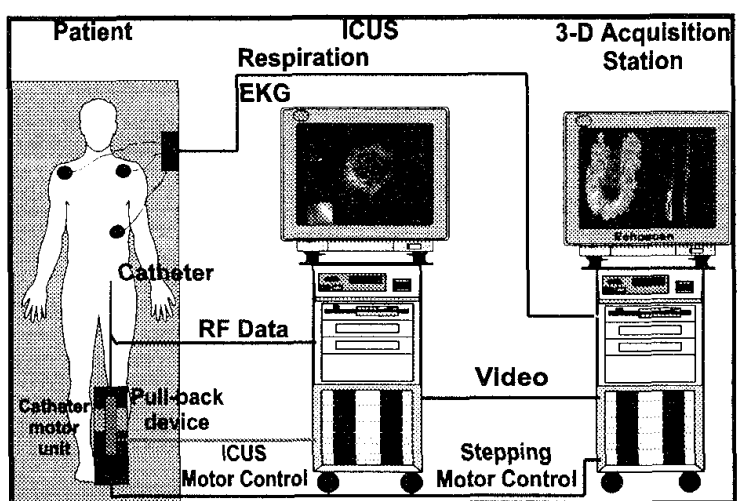

Figure 1

guarantees that pulling a defined distance at the proximal end of the catheter results in an equivalent movement of the tip of the catheter.

The image acquisition and reconstruction station (EchoScan, TomTec, Munich, Germany) receives a video signal input from the ICUS machine and is connected to the patient to monitor the ECG and respiration (impedance method). The acquisition station is also connected to a custom-designed pull-back device (Thoraxcenter, Rotterdam, The Netherlands) to be controlled by the steering logic of the acquisition station, considering heart rate variability and (optionally) the respiration [5].

\subsection{Image Acquisition}

The acquisition station digitises the ICUS images (frames) with $40 \mathrm{~ms}$ intervals to a maximum of 25 images for one heart cycle. It starts with the digitisation after detecting the peak of the R-wave and it acquires the first new available ICUS image on the echo unit (see figure 2).

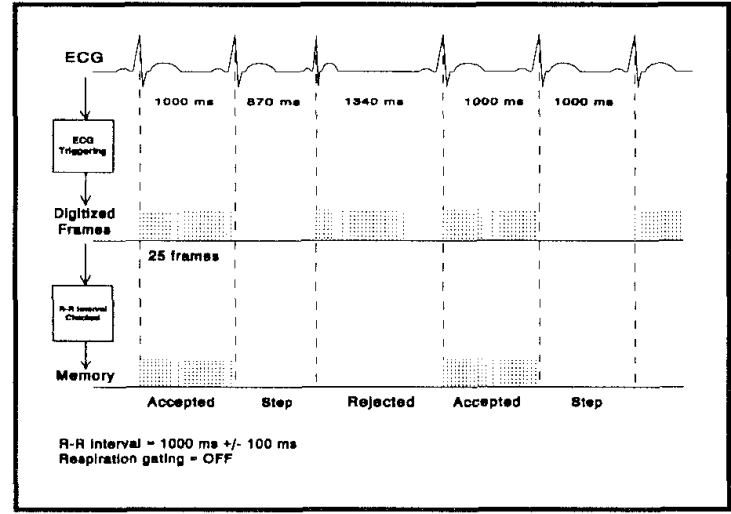

Figure 2

At first the station monitors the ECG and respiration for two minutes and produces two scatter diagrams of the measurements. The software determines the upper and lower limits of the duration of the cardiac cycle and the depth of inspiration/expiration, after which the operator can fine tune these intervals.

After the ICUS images of one heart cycle are acquired the software retrospectively checks if the R-R interval and respiration depth meet the predetermined ranges (see figure 2 ), before the digitised images are stored. Otherwise, the cycle is rejected, removed from the computer memory, and a new sequence at the same transducer position is acquired. After a valid acquisition of one heart cycle the stepping motor receives pulses until the next position on the longitudinal axis is reached. The process is repeated until the end of the longitudinal scan distance is detected. Now the operator must calibrate the echo images for on- and offline quantitative measurements, and the acquired images can be stored on hard-and/or M.O.-disk.

The mean acquisition time to obtain one data set was $3 \pm$ 2 minutes, applying longitudinal intervals of 0.1 to 0.25 $\mathrm{mm}$, an image resolution of $256 * 256$ pixels using 8 bits per pixel, 6 images per R-R cycle, and an average scan length of $4 \mathrm{~cm}$.

\section{Image Processing}

To save time and to speed up the acquisition process the images are stored consecutively in one single data structure. The analysis software used is able to access the images needed for analysis directly from this stack of unprocessed images with respect to their place in the R-R interval

The same data sets are also being used for threedimensional reconstruction's. However, it is necessary to separate the images and to position them to their correct place in the R-R interval for this purpose. During this postprocessing phase the images are formatted in volumetric data sets $(256 * 256 * 256$ pixels/each 8 bits $)$, as can been seen in figure 3 . During this process the images are being filtered and the vessel contours are smoothened.

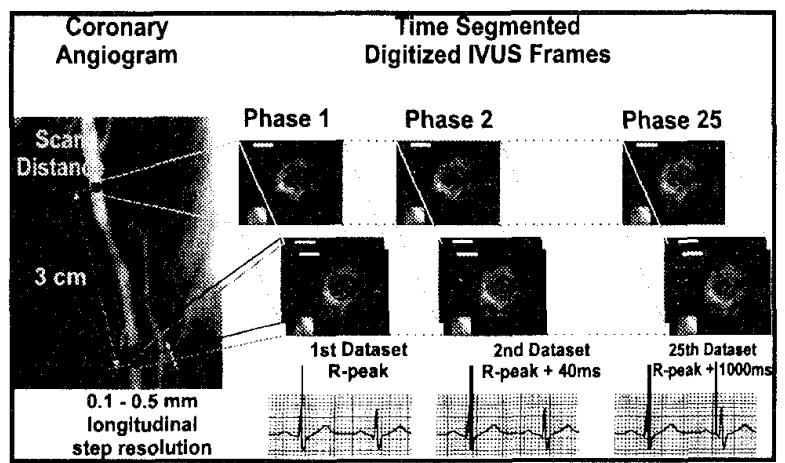

Figure 3 


\subsection{On- and Off-line Analysis}

At the Thoraxcenter an in-house produced Semiautomated contour detection program for cross-sectional and volumetric quantification of 3-D ICUS data sets is used $[6,7]$. This program will be integrated into the software of the 3-D image acquisition system. Due to the fact that the 3-D image acquisition software runs under DOS and the analysis software under windows, rebooting of the machine is momentarily necessary. After the initiating sequence to start the analysis software the raw ICUS images can be loaded into the computer memory with a maximum limitation of 200 images. It took $9 \pm 3$ minutes for an experienced operator to perform an on-line ICUS analysis of a coronary segment with typical acquisition parameters as mentioned in paragraph 2.1 .

\subsection{Clinical Results}

Two comparison studies have been carried out to see if the described approach results in more accurate vessel dimension analysis. Coronary vessel analysis of ICUS image data sets acquired ECG-triggered vs. Non-triggered were compared and dynamic vessel dimension changes over the R-R interval within the same coronary segment were investigated. In figure 4 longitudinal cross-sections of a Non-triggered acquisition of a coronary segment can be seen.

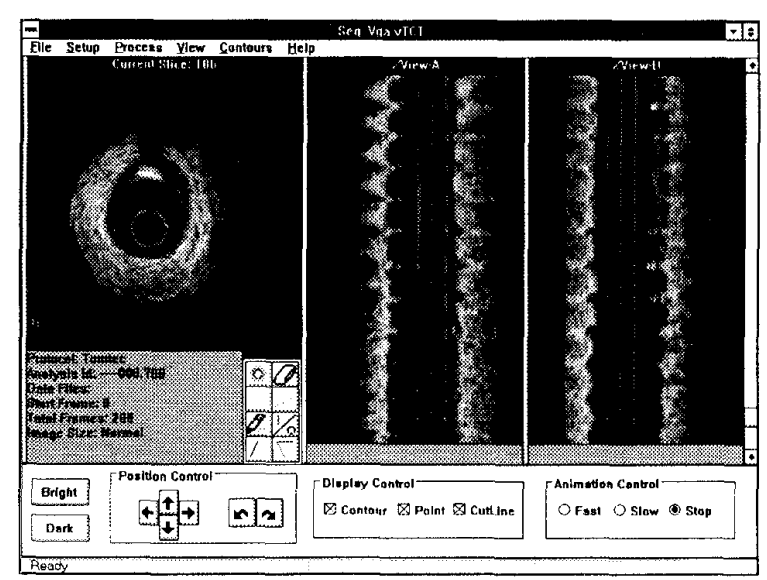

Figure 4

It is clear in this figure that in some parts of this coronary segment it is difficult to point out where exactly the luminal border is. However for a true perception the images should be viewed on a high resolution monitor instead of the limited space and quality of printed images.

Figure 5 shows a coronary segment from the same patient but now ECG-Triggered.

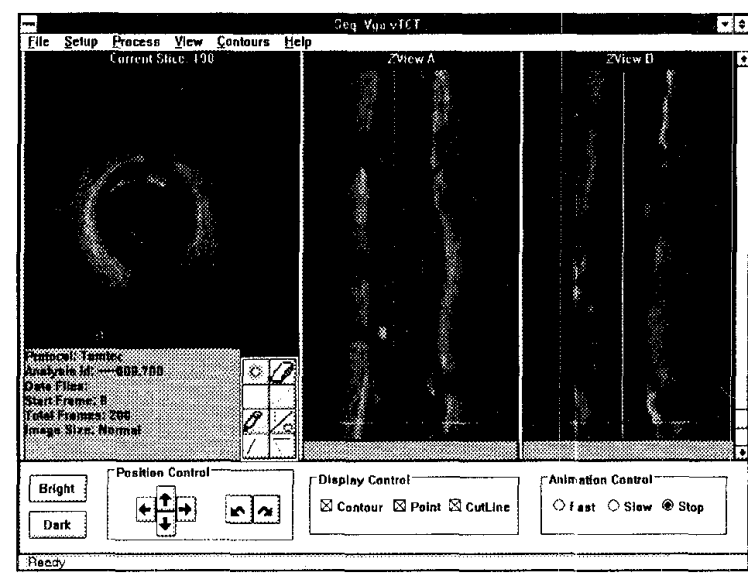

Figure 5

In this image it is better visible where the luminal border is. The sharper defined edges and the lack of motion from left to right of the vessel wall helps to decrease the analysis time into half the time needed for non-triggered data sets. This is mainly due to the fact that less manual interactions to correct the automated found contours are necessary.

For the volumetric quantification study 28 angiographally straight atherosclerotic segments during 19 diagnostic and 7 procedures after coronary intervention (26 patients; 21 male; $54.9 \pm 9.1$ years) were examined. Segments were located in the left anterior descending (15), right (11) and circumflex (2) arteries. At a maximum rate of 5 images per $\mathrm{mm}$ a total of $110 \pm 39$ images per segment was sampled (40 ms after the R-top) and digitised (48-194 images/segment; corresponding length : 9.6-38.8 mm). The results showed a high intraobserver (IAO) and interobserver (IRO) reproducibility : the mean values (delta) ranged from $0.14 \%$ to $1.51 \%$. Earlier studies using non-triggered data sets digitised off-line from video tape showed variation coefficients for lumen volume of $3 \%$ and for plaque volume of $1.8 \%$ [7].

The study for dynamic changes in vessel dimensions was performed in 13 patients after catheter-based coronary intervention. Longitudinal step resolution varied from 0.1 to $0.25 \mathrm{~mm}$ (ea. From 10 down to 4 images $/ \mathrm{mm}$ ), the scan length varied from 2 to $5 \mathrm{~cm}$. The comparison of the enddiastole (ED) and the end-systole (ES) volumetric analysis showed no significant difference in the mean values of vessel dimensions, but showed a large standard deviation of the difference (delta) : Luminal volume : $160 \pm 151 \mathrm{~mm}^{3}$ (ED) vs. $162 \pm 159 \mathrm{~mm}^{3}$ (ES), $-2 \pm 8 \mathrm{~mm}^{3}(\Delta)$; Plaque volume : $117 \pm 101 \mathrm{~mm}^{3}$ (ED) vs. $118 \pm 96 \mathrm{~mm}^{3}$ (ES), -0.8 $\pm 5 \mathrm{~mm}^{3}(\Delta)$; Luminal area : $6.5 \pm 3.9 \mathrm{~mm}^{2}(\mathrm{ED})$ vs. $6.6 \pm$ $4 \mathrm{~mm}^{2}$ (ES), $-0.06 \pm 0.38 \mathrm{~mm}^{2}(\Delta)$. So on average it seems that systolic-diastolic changes of vessel dimensions are smoothened out, but in individual cases a large difference in vessel dimensions can be found. This indicates that for 
research purposes in which high accurate vessel analysis is necessary, such as pro/regression studies, the described method could maybe provide higher accuracy than the current widespread used techniques.

\section{Limitations}

The proposed technique suffers from the following limitations :

- No spatial movement information of the echotransducer is currently registered (ea. straight pipe representation).

- The resolution of the ICUS transducers determines the upper resolution limit of 3-D reconstruction's and analysis.

- The acquisition time is longer compared with uniform velocity pull-backs.

- The technique is more costly than the currently widespread used techniques.

\section{Future Developments}

The most important improvement could be found in registering the spatial movement of the echotransducer during a pull-back sequence, ea. tracking the tip of the catheter. This could provide also true length measurements. Work on this subject has been carried out at the Thoraxcenter [8]. This method works momentarily with a very time consuming technique of off-line digitisation of the spatial movement of the cathetertip from BI-plane angiographic films. Currently work is under construction to produce an image acquisition station which can acquire BIplane angiographic images simultaneously with ICUS images. This could be a major improvement of speeding up the process to reconstruct a coronary segment with his true curvature.

\section{Conclusion}

The feasibility of ECG-gated pull-backs for producing accurate analysis of vessel dimensions could be demonstrated. High-quality ICUS data sets were obtained, not showing cyclic saw-shaped artifacts as seen by nongated approaches. Therefore this method is warranted in progression/regression of atherosclerosis studies.

\section{References}

[1] Roelandt JRTC, Di Mario C, Pandian NG, Li W, Keane D, Slager CJ, de Feyter PJ, Serruys PW. Three-dimensional reconstruction of intracoronary ultrasound images: rationale, approaches, problems, and directions. Circulation 1994; 90:1044-1055.

[2] Karson TH, Zepp RC, Chandra S, Hill RM, Waitz AS, Patten JP, Obuchowski NA. European Heart Journal 1996; volume 16 (abstract supplement): 270.

[3] Di Mario C, von Birgelen C, Prati F, Soni B, Li W, Bruining $N$, de Jaegere PJ, de Feyter PJ, Serruys PW. Three-dimensional reconstruction of intracoronary ultrasound: clinical of research tool? Br Heart J 1995; 73 (suppl. 2):26-32

[4] von Birgelen C, Di Mario C, Prati F, Bruining N, Li W, de Feyter PJ, Roelandt JRTC. Intracoronary ultrasound: Three-dimensional reconstruction techniques. In: de Feyter PJ, Di Mario C, Serruys PW, eds. Quantitative coronary imaging. Rotterdam: Barjesteh, Meeuwse \& Co. 1995: 181197.

[5] Bruining N, von Birgelen C, Di Mario C, Prati F, Li W, Den Hoed W, Patijn M, de Feyter PJ, Serruys PW, Roelandt JRTC. Dynamic Three-dimensional Reconstruction of ICUS Images Based on an ECG-Gated Pull-Back Device. In: Computers In Cardiology 1995. Los Alamitos : IEEE Computer Society Press, 1995:633-636.

[6] $\mathrm{Li} \mathrm{W}$, von Birgelen $\mathrm{C}$, Di Mario C, Boersma $\mathrm{E}$, Gussenhoven EJ, van der Putten N, Bom N. SemiAutomatic Contour Detection for Volumetric Quantification of Intracoronary Ultrasound. In: Computers In Cardiology 1994. Los Alamitos : IEEE Computer Society Press, 1994:277-280.

[7] von Birgelen C, Di Mario C, Li W, Camenzind E, Ozaki Y, de Feyter PJ, Bom N, Roelandt JR'TC. Volumetric quantification in intracoronary ultrasound: validation of a new automatic contour detection method with integrated user interaction. Circulation 1994; 90: I-550.

[8] Laban M, Oomen JA, Slager CJ, Wentzel JJ, Krams R, Schuurbiers JHC, den Boer A, von Birgelen C, Serruys PW, de Feijter PJ. ANGUS: A New Approach to ThreeDimensional Reconstruction of Coronary Vessels by Combined Use of Angiography and Intravascular Ultrasound. In : Computers in Cardiology 1996. Los Alamitos : IEEE Computer Society Press, 1995:325-328.

Address for correspondence.

Bruining N.

Thoraxcenter, University Hospital Rotterdam Dijkzigt

Room BD308b

Dr. Molewaterplein 40

3015 GD Rotterdam

The Netherlands

Tel.:(31)104635334

Fax.:(31) 104634444

E-mail : bruining@thch.azr.nl 